\title{
Le comique « avorté » de Vassilii Sigariov
}

\section{Tania Moguilevskaia}

\section{OpenEdition}

\section{Journals}

Édition électronique

URL : http://journals.openedition.org/recherchestravaux/300

DOI : 10.4000/recherchestravaux.300

ISSN : 1969-6434

\section{Éditeur}

UGA Éditions/Université Grenoble Alpes

\section{Édition imprimée}

Date de publication : 15 octobre 2006

Pagination : 63-75

ISBN : 978-2-84310-099-4

ISSN : 0151-1874

\section{Référence électronique}

Tania Moguilevskaia, "Le comique « avorté » de Vassilii Sigariov », Recherches \& Travaux [En ligne], 69| 2006, mis en ligne le 30 septembre 2008, consulté le 08 septembre 2020. URL : http://

journals.openedition.org/recherchestravaux/300; DOI : https://doi.org/10.4000/recherchestravaux 300

(C) Recherches \& Travaux 


\section{Le comique «avorté» de Vassilii Sigariov}

La Pâte à modeler , pièce que Vassilii Sigariov a écrite en 2000, à l'âge de 23 ans, est un texte d'une rare noirceur. La pièce raconte le chemin de croix de Maxime, un adolescent de I4 ans, orphelin, qui n'arrête pas de prendre des coups. Au fil d'un parcours sans but apparent, il croise des ivrognes, des SDF, des prostituées, des professeurs hypocrites et indifférents, des voisins violents, des vieilles femmes agressives. Environné d'adultes hostiles et menaçants, sans père ni mère, il ne trouve dans son entourage aucun modèle positif. Il sera trahi par son meilleur ami, renvoyé de l'école, violé par des malfrats et finalement assassiné.

La critique russe de l'époque s'est trouvée choquée par cette œuvre qui, par ailleurs, a été reçue avec enthousiasme par les cercles de la dramaturgie contemporaine et a recueilli de nombreux prix $^{2}$. On pouvait lire cette présentation dans la revue Afisha:

Sigariov a écrit une pièce impossible. C'est une vie d'adolescent traitée comme dans un scénario qui court de l'enterrement de son ami à sa propre mise à mort. Elle est écrite dans la langue qu'on parle à Nijniaia Salda ${ }^{3}$, à Rostov-sur-Don ${ }^{4}$, à Moscou aussi, peut-être, qu'on parle quelque part ainsi, mais en tout cas, pas au thêâtre.

La première - et seule pour l'instant en Russie ${ }^{6}$ - mise en scène de $L a$ Pâte à modeler par Cyril Serebrennikov a révélé le texte au grand public. Elle

I. La traduction française de La Pâte à modeler de M. Zonina et J.-P. Thibaudat est publiée par Les Solitaires intempestifs, coffret "Théâtre contemporain russe», Besançon, 200I.

2. En Russie, La Pâte à modeler a reçu les prix littéraires "Antibooker» 2000 et "Début» 2000. En 2002, Vassilii Sigariov a reçu des mains de Tom Stoppard le prix «Evening Standard» en tant que "Dramaturge le plus prometteur".

3. Dans la région où Sigariov est né.

4. La ville où Kirill Serebrennikov est né.

5. E. Kovalskaia, Afisha, Moscou, 22 avril 2003.

6. La Pâte à modeler a été traduite en de nombreuses langues et montée de multiples fois hors de Russie, notamment en Angleterre (création en anglais en 2002 par Dominic Cooke 


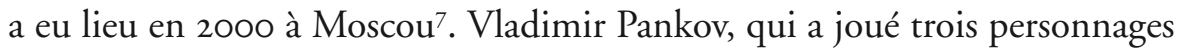
dans cette création et a assuré la composition musicale, exprime le choc qu'il a ressenti à la lecture de la pièce:

J'ai regardé le texte, il était énorme! [...] J'ai dit à Serebrennikov: tu es fou ou quoi? C'est un texte typique de la Perestroïka, quel intérêt de jouer cela aujourd'hui ? C'est de la tchernoukha ${ }^{8}$ pure. ${ }^{9}$

Pour lui, comme pour beaucoup d'autres, la mise en scène de Serebrennikov a pourtant été la révélation d'une nouvelle façon de mettre en scène un texte violent et contemporain:

Cyril Serebrennikov a pris le contre-pied de cette dramaturgie [...]. La Pâte à modeler m'a secoué. J'ai compris qu'on peut mettre en scène un texte brutal d'une manière subtile et belle. Et qu'alors, même les injures sonnent différemment.

Serebrennikov pense que "par le niveau de concentration de violence, d'émotion et de douleur, le texte de Sigariov s'apparente aux meilleurs exemples de l'épopée tragique ${ }^{\mathrm{IO}}$ ". Sa mise en scène a fait ressortir la dimension onirique et poétique du texte et a mis en relief son côté burlesque à travers l'utilisation systématique de la pantomime et de la danse contemporaine.

Du point de vue du mode de fiction, on pourrait rattacher cette pièce à un certain nombre de "détours" dramaturgiques dans le sens que JeanPierre Sarrazac attribue à cette notion ${ }^{\mathrm{II}}$. En passant, en premier lieu, par un des modèles romanesques: celui du roman d'apprentissage dans lequel un "niais" découvre le monde des autres. Le titre initial de la pièce était $L a$ Chute de l'innocence. Cet apprentissage se traduit par une série d' "échecs", échec sentimental, échec scolaire, et se termine brutalement par la mort du héros adolescent. De la part de ses «initiateurs », Maxime apprend la violence et devient victime de celle-ci. Comme dans les romans picaresques, le pauvre hère vit des aventures, explore différents milieux sociaux tout au long d'un

au Royal Court Theatre Upstairs, Londres), au Danemark, en Roumanie, en Allemagne, aux Pays-Bas, en République tchèque. La dernière mise en scène en date, en portugais, est celle de Nino Cardozo au Teatro Carlos Alberto, à Porto, en mars 2006.

7. Au Centre de la dramaturgie et de la mise en scène, sous la direction de A. Kazansev et M. Rotchine.

8. Signifie en russe "noirceur». Ce terme désigne une tendance précise de la dramaturgie russe (Nikolai Koliada, Ludmila Petrouchevskaia) des années 1970-1980 qui traitait de la vie de privation et d'humiliation de l'homme soviétique. Interdites à l'époque, ces œuvres ont été beaucoup jouées après la Perestroïka et ont fini pas lasser le spectateur par leur aspect désespéré.

9. Entretien de T. Moguilevskaia avec V. Pankov, UBU Scènes d'Europe, n² 29, oct. 2003, p. 29.

Io. C. Serebrennikov pense "qu'on ne doit pas faire violence au spectateur qui vient au théâtre", UBU Scènes d'Europe, numéro cité, p. 25.

II. Voir notamment J.-P. Sarrazac, Jeux de rêves et autres détours, Belfort, Circé, 2004. 
itinéraire de vie. La structure lâche du texte est celle d'un récit à épisodes. Bien que Maxime ne se livre pas à travers des monologues et qu'on ne puisse pas parler d'une pseudo-confession, tout au long de la pièce l'auteur semble décrire le monde du point de vue de Maxime.

Sur le plan des détours proprement dramatiques, il s'agit d'un drame itinérant de 33 "séquences", qui se déroulent à chaque fois dans des lieux différents, autant de stations entre lesquelles le personnage principal se déplace "en courant»: ce sont les stations d'un chemin de croix moderne déterminé par la dégradation des normes morales dans la société, dont Maxime fait les frais. Mais La Pâte à modeler est aussi la parabole d'un adolescent qui perd la vie parce qu'il est projeté dans un univers où seule la violence est maîtresse.

Le plan «réaliste» que constituent les stations du parcours quotidien du héros - l'école, les rencontres avec son ami Lekha, etc. - se combine avec une dimension onirique double: les apparitions merveilleuses d'ELLE (séquences 3, I5, 26, 33), et d'autres, macabres, comme l'apparition du fantôme de Spira, le copain de classe décédé qui appelle Maxime à le rejoindre (séquences 9 et I6). Ainsi, la forme du "jeu de rêves" vient enrichir ce drame qui se révèle hybride.

\section{Farce et dérèglements}

L'événement principal qui enclenche la machine d'exclusion de l'école dont Maxime est victime, est une farce grossière, du théâtre dans le théâtre que le héros met en scène lui-même: dans les toilettes des garçons, Ludmila Ivanovna, professeur de langue et littérature russes, venue comme à son habitude traquer les jeunes fumeurs, ordonne à Maxime de se tourner face à elle. Comme il refuse d'obéir, Ludmila Ivanovna le retourne de force et se retrouve devant un énorme phallus en pâte à modeler collé sur le ventre de l'adolescent.

Cette séquence de farce n'est pas la seule de la pièce. N'oublions pas la dimension farcesque du roman picaresque auquel la pièce ressemble tant par le sujet que par son agencement. En effet, un certain nombre de scènes dont Maxime est témoin ou auxquelles il participe sont des farces. Dans la scène du mariage que Maxime croise par hasard sur son parcours, les Jeunes Mariés se livrent à une farce gratuite, délirante, grotesque, en provoquant et attaquant le héros. Ils le tabassent et en rient comme d'une bonne blague. Les Jeunes Mariés, une image associée à l'amour et à la fête, se comportent en vulgaires voyous. Le renversement d'un stéréotype... Les personnages, plus d'une vingtaine dans la pièce, sont des masques, réduits à leur statut, profession ou trait de caractère. D’ailleurs, ils portent rarement des noms: les Jeunes Mariés, le Voisin, la Première et la Deuxième Femme, La Première et la Deuxième 
Vieille, nombre d'autres Femmes et Hommes, Mecs et Filles. Sur plus d'une vingtaine de personnages, cinq seulement ont une identité.

Traditionnellement, la farce repose sur l'affrontement comique de personnages populaires qui cherchent le plus souvent à duper, «farcer» ou dominer autrui $^{{ }^{2}}$. Ne pourrait-on pas regarder Maxime comme une espèce d'imbécile dupé par tout le monde? La duperie déclenche la scène où les Jeunes Mariés décident de s'amuser aux dépens d'un passant innocent; ou encore celle où le Voisin demande ingénument à Maxime de lui prêter un briquet pour lui tomber dessus aussitôt en utilisant ce briquet contre lui. L'Homme du stade dupe une femme en s'esquivant avec la bouteille qu'il avait promis de partager. Natakha promet aux garçons une soirée avec des filles pour les livrer à deux violeurs.

Les sentiments et la psychologie sont réduits à des pulsions primitives. Le bas corporel occupe, comme on le sait, une place première dans la farce. Et les pulsions sexuelles sont ici récurrentes: la Fiancée place de force la main de Maxime sur ses seins et dit être "déjà toute mouillée»; la femme éméchée enfonce la tête de Maxime sous ses jupes pour un cunnilingus contraint; et la grand-mère de Spira parle de sa fille, la mère de Spira, en disant qu'il faudrait «enlever les utérus des mères comme elle».

Autre élément récurrent: l'alcool fait littéralement courir les personnages, dicte leurs actions. La mère du défunt court après le bus pour pouvoir boire au repas funéraire. L'ayant raté, elle change aussitôt de direction pour courir après un autre coup à boire, promis par un inconnu depuis une fenêtre. Une autre femme, attirée par la bouteille de vodka, suit dans un stade le premier venu. L'alcool est un manipulateur en puissance, une source d'embrouilles, de scandales, d'humiliations et d'autohumiliations, d'écarts moraux... L'alcool bestialise les personnages en les jetant littéralement «à quatre pattes».

L'éthylisme ambiant que Sigariov décrit n'est pas pour autant exagéré. On doit tenir compte du fait qu'une fois remise dans son contexte russe, La Pâte à modeler décrit un état réel des choses. Confrontée à un chômage massif dû au déclin de l'industrie, sa ville natale, Verkhniaia Salda (50 ooo habitants) bat, depuis une dizaine d'années, les records statistiques d'empoisonnements dus à la consommation d'alcool frelaté, ainsi que le record du niveau de délinquance lié à l'alcool et la drogue. Dans une autre pièce de Sigariov, Le Lait noir, la caissière d'une station ferroviaire fabrique et vend de la vodka en expliquant ainsi l'alcoolisme généralisé de la population:

[...] ces parasites boivent comme des trous [...]. Mais si on y réfléchit, que peuvent-ils faire d'autre? Pas de cinoche. Pas de télé non plus, on est dans une zone où les ondes ne passent pas, c'est une fosse ici. Voilà pourquoi ils boivent.

I2. Voir l'article "Farce» du Dictionnaire encyclopédique du théatre, sous la dir. de M. Corvin, Paris, Larousse, 2003, p. 628. 
Fait tragique, l'alcoolisme constitue en même temps une source inépuisable de comique car il transforme, travestit les êtres. C'est une force qui manipule les personnages qui se conduisent comme des pantins, des marionnettes. Nous sommes dans l'une des variations du "mécanique plaqué sur le vivant» bergsonien.

La violence verbale et physique est omniprésente dans la pièce, comme nous le voyons dans le répertoire d'actions citér. ${ }^{13}$ C'est une des thématiques principales de la pièce. Dirigée contre Maxime, mais aussi contre d'autres personnages qui maintiennent entre eux des rapports sadomasochistes, brutaux et cruels, la violence engendre un cercle vicieux où Maxime est obligé de répondre par la violence aux agressions, ce qui le conduit à la mort. Sigariov multiplie les scènes et enchaîne les séquences et les microséquences dans lesquelles la violence se manifeste, jusqu'à provoquer une saturation et une réaction d'écœurement chez le spectateur. Cependant, grâce à l'exagération et à la multiplication de telles scènes, et grâce à leur côté grotesque, la violence tourne à la dérision. On pourrait dire, en suivant Jean Emelina, que nous avons du mal à partager les tourments des personnages "parce qu' ils sont dits très vite et non pas dépeints ${ }^{\mathrm{I}}{ }$. La fréquence incroyable des actes violents, le déferlement des «mauvaises nouvelles» ne permet pas de s'y arrêter et devient une source de comique burlesque.

L'auteur peut faire rire, enfin, en évoquant la situation économique des personnages. Une mère évite l'exclusion de son fils en corrompant le professeur avec un abonnement à la piscine (inaccessible et tant convoité à l'époque soviétique). Une pauvre ivrogne réclame l'amour du premier venu en lui faisant des promesses de richesses: «Tu m'épouseras? Mon papa c'est le directeur d'usine. Il t'offrira une bagnole. Une Mercedes. Et un cinq pièces. Tu m'épouseras?» (p. 53) Les biens matériels sont tellement rares dans cet univers de pauvreté que leur évocation a quelque chose d'irréel et d'insolite, ce qui produit également le comique.

I3. Voici le répertoire non exhaustif d'agressions verbales et physiques subies par Maxime et les autres personnages:

Maxime se fait chasser par des vieilles menaçantes de l'appartement du défunt qui le traitait de pervers sexuel (séquence 2). La mère du copain défunt de Maxime se fait chasser de l'enterrement et insulter par sa propre mère (séq. 3). Lekha viole Maxime (séq. 6). Les deux Jeunes Mariés tabassent Maxime et l'abandonnent (séq. 8). La professeur de russe insulte la grand-mère de Maxime (séq. I3). Maxime se fait insulter et tabasser par la bande des copains de Lekha (séq. I4). Maxime est agressé verbalement et physiquement par le voisin. Maxime allume un incendie devant la porte de ce dernier (séq. 17). Une Inconnue ivre se fait taper dessus par un partenaire sexuel occasionnel et essaye elle-même de violer Maxime (séq. I8). Maxime et Lekha sont humiliés et violés par deux ex-taulards (séq. 22). Maxime est menacé par le voisin (séq. 3I). Maxime revient se venger chez les violeurs. On lui écrase la main avec la porte (séq. 32). Les violeurs tabassent Maxime et essayent de le jeter par la fenêtre (séq. 33).

14. J. Emelina, Le comique. Essai d'interprétation générale, Paris, Sedes, 1996, p. 6I. 
Sigariov avoue les origines autobiographiques de sa pièce:

La fable de La Pâte à modeler est constituée de petits fragments tirés de différentes histoires. Une séquence d'événements qui sont arrivés à différentes personnes: à moi, à mon frère, à mes amis [...]. J'ai assisté à l'enterrement d'un gars de mon école qui s'est pendu. [...]. La vie de Maxime, je ne l'ai pas inventée, j'ai assemblé des biographies, parmi lesquelles la mienne. [...] Mon institutrice est encore fâchée contre moi parce que j'ai écrit cette pièce. Le pénis en pâte à modeler a bien existé. Je l'ai sculpté quand j'étais étudiant à l'Institut pédagogique. Mon petit frère se plaignait tout le temps que son institutrice venait dans les toilettes des garçons. J'ai donné ce pénis à mon frère et il l'a sorti de son pantalon devant la prof. On l'a viré de l'école. Je me sens assez coupable, surtout que depuis, mon frère n’a pas choisi la meilleure voie, il est devenu toxico. Aujourd'hui, il purge une longue peine de prison. ${ }^{15}$

Je n'invente pas mais j'essaie de rendre les choses plus attractives, plus aiguës, ou bien je les développe dans une direction précise, tout en me basant sur une histoire réelle qui maccroche. ${ }^{16}$

Il s'agit alors d'une écriture partant de l'étude du réel, mais délibérément outrée, teintée de noirceur. À la question de cette noirceur qu'on perçoit chez Sigariov, il répond qu' «il faut bien que quelqu'un s'en charge. Le théâtre ne peut pas être qu'un divertissement. Noirceur égale véritét7 ${ }^{17}$.

\section{Effets de situations et langage des corps}

L'extravagance des situations, comme celle où les Jeunes Mariés tabassent le premier venu, renverse les clichés habituels. Un sujet noble est traité de manière grossière, vulgaire. La Mariée est loin d'être une jeune femme gracieuse et aimante comme on pourrait le penser. Elle drague le passant sous les yeux de son époux et lui propose de partir avec elle. Rejetée, elle s'en prend à Maxime, le bat et encourage son époux à faire de même. Ils laissent Maxime en plan et rejoignent leurs invités en riant. La situation "noble» est traitée ici sous forme parodique. Les Mariés se comportent comme une bande d'adolescents hyperviolents et écervelés.

Une autre parodie est celle de la «scène d'amour» qui se déroule dans les gradins d'un stade désert. C'est ici que se rendent deux personnes qui viennent de se rencontrer: une trentenaire éméchée et un jeune homme qui a entraîné cette dernière grâce à la promesse d'une bouteille de vodka. La situation tourne au malentendu: la femme tient un discours de femme amoureuse qui ne s'accorde guère avec la situation:

15. M. Raikina, "Un néophyte de pâte à modeler», Moskovskii Komsomolets, Moscou, 3 décembre 2002.

16. Entretien de V. Sigariov avec I. Alpatova, Kultura, 30 octobre - 5 novembre 2003.

17. M. Raikina, «Un néophyte de pâte à modeler», art. cit. 
FEMME: Embrasse-moi.

GARS: J'ai envie...

FEMME : Je suis belle?

GARS: Oui, belle.

FEMME: Tu m'aimes?

GARS: Je t'aime.

FEMME: Moi aussi. Comment tu t'appelles?

GARS: Dima... Je t'en prie...

FEMME: Tu m'épouseras?

GARS: Je t'épouserai. Je t'en prie...

FEMME : Allez, vas-y.

Un autre renversement de cliché: Ludmila Ivanovna est professeur de langue et de littérature russes, fonction à laquelle est associé le cliché d'humanisme, mais elle s'acharne sur un élève, le traitant de "salopard», elle parle de lui comme d'un chiot dont l'école ferait mieux de se débarrasser dans l'espoir qu'il se fasse «buter dans la rue [...]. Une ordure de moins sur terre». Le même professeur adopte une posture grossière par rapport à la vieille et impuissante grand-mère de Maxime. L'accusation dont celle-ci fait l'objet de la part de l'enseignante est absurde et risible puisque lui est reproché son manque de responsabilité en tant que tutrice. Surtout si on tient compte de l'état des orphelinats en Russie:

LA PROF: Alors, il fallait pas le prendre sous tutelle. [...] C'est une énorme responsabilité, chère madame.

GRAND-MÈRE: Mais où est-ce qu'il pourrait aller...

LA PROF: Notre État, Dieu merci, a créé toutes les conditions. Et il y en a des comme vous qui n'arrivent toujours pas à comprendre. Et n'en font qu'à leur tête. Le résultat le voilà [...], des drogués, des salopards et des tas de rebuts.

C'est dans une classe décorée avec les portraits des grands auteurs classiques russes $^{18}$ que le professeur se laissera facilement corrompre. En échange d'un abonnement à la piscine et d'une promesse de faux témoignage, elle oubliera la présence de Lekha dans les toilettes pour accuser Maxime et obtenir son exclusion. Le comique provient du décalage de la situation dans une autre scène où Maxime est injustement accusé. Il est venu rendre un dernier hommage à son copain défunt Spira; en essayant de s'approcher du cercueil à travers la foule, il se fait traiter de pervers sexuel par une Vieille. Par ailleurs, le procédé de travestissement, de bestialisation de l'humain est utilisé dans la scène où la femme éméchée drague Maxime:

Elle voit Maxime.

L'enlace.

I8. En URSS, dans chaque classe de littérature et de langue russes, étaient accrochés les portraits de Tolstoï, Dostoïevski, etc. 
L'embrasse sur le visage.

Retrousse sa jupe.

Enfonce le visage de Maxime contre son slip déchiré.

Maxime dégueule sur elle.

La femme le repousse.

Elle ramasse sur lui un morceau du pain mal digéré. Le fourre dans sa bouche.

Dans un récent entretien, l'auteur notait que «les paroles peuvent être remplacées par les gestes et vice-versa»:

Pour ma part, je n'aime pas quand on parle beaucoup dans une pièce. Tout doit être communiqué entre les lignes, dans les gestes. Si on se mettait à tout dire à haute voix, cela ne m'intéresserait pas du tout. ${ }^{19}$

La gestuelle burlesque est en effet très présente dans la pièce. Elle met en scène les corps comiques, «[...] le corps est la matière première du rire, le corps muet des burlesques, le corps individuel, mais aussi le corps collectif ${ }^{20}$ ".

La scène de mariage commence par la scène où "Le Fiancé, en noud papillon, est assis sur une poubelle, devant l'entrée de la salle des fêtes. La Fiancée sur ses genoux.» (p. 30) Ensuite s'enchaînent des actions:

La Fiancée aperçoit Maxime

Jette sa cigarette

Murmure quelque chose à l'oreille de son fiancé

Descend d'un bond de ses genoux.

Interpelle Maxime

Demande une cigarette

S'avance vers lui en retroussant sa robe

Attrape une main de Maxime et la plaque contre sa poitrine

Maxime arrache sa main, s'en va

Le Fiancé part d'un bond à la poursuite de Maxime

Le Fiancé [...] donne un coup de poing dans le visage, des coups de pieds,

La Fiancée rit aux éclats.

Enlève une chaussure, frappe Maxime

Crache sur le visage de Maxime

Les deux Jeunes Mariés rigolent et partent en courant.

Les gestes sont stylisés pour signifier l'action sans obligation de recourir aux dialogues. Dans la scène du stade entre la Femme éméchée et le Gars à la bouteille, on retrouve la même gestuelle outrée et évidente, qui réduit la situation à un schéma de duperie grossière. L'auteur pousse ses personnages à jouer toute une séquence burlesque dans la scène didascalique où Maxime observe les gens en bas du toit. Il s'agit d'une foule de personnages non identifiés et ne participant pas à l'action principale:

19. Entretien de T. Moguilevskaia avec V. Sigariov, avril 2006.

20. O. Mongin, Éclats de rire. Variations sur le corps comique, Paris, Seuil, 2002, p. I2. 
Telles des fourmis, en bas, les gens grouillent. Ils vont faire leurs affaires, ils sont en retard. Se saluent et se disent adieu aussitôt. Lancent des mégots dans les poubelles, mais ratent leur coup. Se racontent des blagues et rigolent d'eux-mêmes. Trébuchent du pied gauche et crachent par-dessus l'épaule gauche. Trébuchent du pied droit et sourient. Se mouchent le nez entre leurs doigts et marchent sur leur morve. Trouvent des kopecks et perdent des roubles. Courent pour rattraper un bus, mais le ratent. Ils se rencontrent et se quittent. Se réjouissent et s'attristent. Aiment et hä̈ssent. (séquence 27)

Le regard de l'auteur sur l'humanité est à la fois ironique et empathique dans cette scène où les figurants "tournent en rond", multiplient des comportements "mécaniques», des ratages, des actes de distraction et d'autres actions contradictoires.

Sigariov utilise l'expression "scènes muettes " pour définir la scène de farce jouée à la professeur de russe "Il tient, collée contre sa braguette, une énorme bite en pâte à modeler. Luisante et très naturelle. Scène muette» (séquence II, p. 37). La signification de «scène muette» en russe est particulière. Elle apparaît dans la didascalie de la dernière scène du Revizor de Gogol. La société, qui s'est démenée pour accueillir comme il le doit le Réviseur, apprend que c’était un imposteur et que le vrai Réviseur est sur le point de débarquer dans la ville. Surprise générale, consternation : tout le monde se fige dans la posture qui était la sienne au moment de l'annonce. Dans les scènes muettes, les personnages se figent d'un seul coup, comme paralysés. Dans le fragment d'une séquence également liée à une surprise, Lekha "se fige» en apercevant les deux bandits tatoués au seuil de l'appartement de Natakha.

À l'intérieur des séquences de la structure générale, constituée d'épisodes aux liens assez lâches, Sigariov pratique des découpages en séquences plus courtes basées sur des enchaînements très rapides, comme lorsque ces personnages courent tour à tour pour attraper un bus: "Il [...] court pour attraper le bus. La deuxième femme se lève et court à son tour jusqu'au bus. Mais les portières se referment devant son nez. [...] Elle se met à marcher, dans le même sens que le bus.»

Toute une série de solos avec un objet, de duos comiques et de scènes collectives se succèdent dans la pièce, à travers lesquelles le burlesque déroule ses gags. Il est de règle dans ce texte de multiplier les enchaînements comme par exemple dans la séquence 8 : aussitôt après le tabassage de Maxime par les deux Fiancés qui fuient, une voiture passe d'où quelqu'un jette une bouteille de bière vide. Maxime ramasse la bouteille, menace la Fiancée derrière la vitre de la cantine, abandonne son idée et jette la bouteille dans la fenêtre d'un appartement. 


\section{Le comique sigariovien}

«En général mes comédies ne sont pas des réussites. Je crois que l'humour doit être fin et intellectuel. Et moi, je ne sais pas le faire.» Malgré le doute que l'auteur éprouve sur sa capacité à manier la matière comique dans les paroles, il maîtrise parfaitement le dialogue comique. Le procédé principal est la "stichomythie». La stichomythie tend à précipiter le tempo du dialogue en juxtaposant des répliques brèves de même longueur qui se livrent à un duel - ou duo - verbal ${ }^{21}$. Ce procédé détermine le rythme du dialogue sigariovien, brusque et cinglant. L'écriture de ses répliques reproduit une langue parlée et familière, truffée d'injures, syncopée, à l'image de la violence des rapports entre les personnages. Cette langue est utilitaire, servant uniquement à régler les besoins primitifs de ces créatures: faim, soif, défense de son territoire, etc. La langue sert aussi à se protéger ou à blesser l'autre. Mais ces dialogues, en apparence limités dans leur pouvoir d'expression, sont magnifiquement rythmés, presque musicaux. Et les injures et les grossièretés sont autant d'accents qui scandent cette musique du dialogue sigariovien.

L'effet comique vient souvent de la rupture, procédé dominant de ces échanges syncopés où la forme l'emporte sur le contenu: l'inconsistance du dialogue disparaît derrière sa brusquerie, toute en ripostes et en ruptures. Voici un dialogue entre la mère du copain défunt de Maxime et la grand-mère qui l'a apparemment élevé. Cette scène se réduit effectivement à un duel verbal pour la possession des objets du défunt:

DEUXIÈME FEMME: J'embarque ses frusques?

PREMIÈre FEMME: C'est ça! Pour les boire. J'ai des petits-enfants, moi. Ce sera pour eux.

DEUXIÈME FEMME: Et moi, alors, je n'existe pas?

PREMIĖRE FEMME Que dalle.

DEUXIĖME FEMME: Je suis une mère!

PREMIÈRE FEMME T'es une merde comme mère! Les mères comme toi, primo il faut leur enlever l'utérus.

Autre exemple: cet échange de répliques correspondant à la situation où des badauds interrogent Maxime sur la personnalité du défunt.

FILLE: C'est qui qu'on enterre?

MEC: Sais pas. Un cave qui s'est pendu à cause d'une nana. C'est ce qu'on dit. FILLE: Tu déconnes?

MEC: J'te dis, j’sais pas. (

MAXIME: Spira.

MEC: Qui ça?

2I. É. Eigenmann, Méthodes et problèmes. Le mode dramatique, Département de français moderne, université de Genève, 2003. 
MAXIME: Un mec.

MEC: Quel mec? Qui ça?

MAXIME: Personne, comme ça?

MEC: Comme ça, quoi?

MAXIME: Mort.

On peut se demander si l'intention de l'auteur dans les scènes qu'on considère comme comiques est véritablement satirique. «La satire [...] se charge de fureur $[. .$.$] et chasse le rire { }^{22}$." La satire, il est vrai, «bien que son comique soit fondé sur la caricature, s'appuie sur un fond réaliste d'étude des situations et des problèmes quotidiens ${ }^{23} »$. Nous avons vu que cette attitude correspond à celle de Sigariov qui décrit les vices principaux de son entourage, voire de son pays, notamment l'alcoolisme.

Cependant, la satire est censée entretenir avec l'objet qu'elle met en scène un rapport de supériorité, la posture qui induit «le point de vue hautain de l'auteur satirique, son regard en surplomb sur la réalité, la distance qu'il cultive par rapport au monde ${ }^{24} »$. Dans le cas de La Pâte à modeler, malgré la dureté du traitement dégradant que Sigariov réserve à des personnages comme Le Fiancé et La Fiancée, La Femme éméchée, Le Gars du stade et d'autres, en les soumettant à une caricature grossière et souvent repoussante, le lecteur ressent la relative tendresse que l'auteur éprouve à l'égard de ces êtres perdus.

Le comique grossier et simplificateur, farcesque, se transforme et acquiert souvent une dimension tragique grâce aux éléments qui viennent compléter et compliquer notre vision de la situation. Derrière la caricature, on voit se profiler un malaise général de ces êtres, victimes de leur condition... L'auteur entretient avec ses personnages "comiques» une relation ambiguë, entre la critique et la compassion. Ce qui empêcherait l'auteur d'adopter une posture critique par rapport à ces personnages, c'est la conscience qu'il a, apparemment, d'appartenir au même monde qu'eux, de leur ressembler. La fameuse distance qui est une des conditions du rire se trouve systémiquement compromise.

Il existe d'ailleurs un certain malentendu entre l'auteur et la plupart de ses metteurs en scène, notamment en Russie. À plusieurs reprises, Sigariov a exprimé son insatisfaction concernant les mises en scène de ses pièces: "J'ai déjà vu plusieurs mises en scène qui ne parlaient pas de ce sur quoi j'avais écrit [...]. Les metteurs en scène montent le spectacle d'un point de vue politique, alors que moi j'ai écrit sur des êtres humains.»

Il s'insurge contre le propos critique qu'on essaie de lui attribuer sur «la Russie affamée et malheureuse» ou bien sur "des provinciaux démunis et

22 J. Emelina, Le Comique..., op. cit., p. 4I.

23. T. Moguilevskaia, "La satire», dans Poétique du drame moderne et contemporain, Études théatrales, $\mathrm{n}^{\circ}$ 22, Louvain-la-Neuve, 200I, p. II4.

24. J.-P. Sarrazac, L’Avenir du drame, nouvelle édition, Belfort, Circé/Poche, 1999, p. I74. 
miséreux" abrutis par l'alcool. À de nombreuses reprises, il défend ses personnages dans ce qu'ils ont de décalé du point de vue moral, en avouant la complexité des relations qui existent entre ces êtres qui sont, selon ses propres termes, loin d'être parfaits.

Selon André Jolles, «la satire est une moquerie qui porte sur l'objet qu'on réprouve et qui nous est étranger. Nous nous refusons à avoir rien de commun avec l'objet de ce blâme, nous nous y opposons brutalement, nous le dénouons sans sympathie ni compassion ${ }^{25}$ ». Mais Jean-Pierre Sarrazac souligne la difficulté que rencontre aujourd'hui l'œuvre satirique de «la présence de l'Autre en nous-mêmes». Selon lui, à l'époque actuelle, l'auteur ne peut plus stigmatiser les comportements qu'il considère comme extérieurs à lui-même sans tomber dans une sorte de rapport schizophrène au monde, dans une "contradiction de l'extériorité intériorisée ${ }^{26}{ }_{\text {". }}$

Dans les scènes comiques de La Pâte à modeler, il s'agit alors probablement d'un constat, plus que d'une satire. D'un constat à trait forcé qui peut en lui-même constituer une charge contre la situation sociale qui provoque les situations et les comportements décrits. Car en nous livrant ces personnages "à quatre pattes» (c'est souvent le cas dans la pièce), l'auteur n'omet pas de disséminer des indices permettant de reconstituer les conditions sociales dans lesquelles ils évoluent: la pauvreté, l'abandon.

Il est souvent question des immeubles délabrés "typiquement russes", tous identiques avec «la porte d'entrée suspendue à un seul gond», qui se dressent «au milieu des petites baraques en bois», dont l'entrée pue comme à "Buchenwald $»^{27}$. Les équipements sont minables, comme la cantine où le mariage est fêté. Les Jeunes Mariés fument une cigarette assis sur une poubelle. Tout l'univers de la pièce a l'apparence d'une cité à l'abandon où la déshumanisation est à l'œuvre. Une destruction inéluctable soulignée par le trait d'humour noir qui évoque l'espace concentrationnaire.

La Pâte à modeler n'appartient en aucun cas prioritairement au genre comique. Si la veine comique y est très présente, elle ne fait que souligner l'atrocité des conditions de vie et l'horreur des rapports entre les gens. L'élément comique farcesque s'y trouve en tension avec d'autres nettement dramatiques, voire tragiques. Plus précisément, c'est la dimension tragique qui s'impose: l'homme est broyé par des événements, des forces qui le dépassent.

25. A. Jolles, Formes simples, Paris, Seuil, 1972, p. 203, cité par J.-P. Sarrazac, ibid., p. I76.

26. J.-P. Sarrazac, ibid., p. 177.

27. Un pur exemple d'humour noir à la russe: du fait que dans l'enseignement scolaire, les thèmes de la Victoire du peuple soviétique dans la Grande Guerre constituaient un des sujets les plus mis en valeur, la réaction de la plupart de jeunes a été d'en faire un objet de moquerie. Les clichés officiels sont entrés dans le langage comme des expressions idiomatiques. 
Emprisonnés à vie dans des environnements glauques et inhumains, les personnages entretiennent entre eux des rapports sadomasochistes. Les parents déclassés, désespérés et impuissants se confrontent à des enfants en perdition dans un contexte de promiscuité, de crise de l'éducation, de perte des valeurs et de désordre public... C'est un destin collectif qui se dessine, celui, bien réel, de la population démunie des petites villes de la lointaine province russe en proie aux industries en ruine: chômage chronique, salaires ridicules, alcoolisme dévastateur, pauvreté sans espoir...

Le comique vient ici en équilibre du tragique et tend vers l'humour noir. On atteint avec lui les limites du supportable. Un humour où le sourire manque de gaieté, mais qui constitue en quelque sorte le seul recours contre le désespoir ambiant. Proche du carnavalesque et du grotesque, c'est un rire profanateur, transgressif et subversif, qui est un moyen d'assumer l'angoisse existentielle, les coups du sort, en les vidant de leur charge affective ${ }^{28}$. En mettant ainsi à distance les émotions et les passions, il est possible de continuer à vivre.

28. Voir l'article "Humour noir» de P. Jallageas dans le Dictionnaire international des termes littéraires, en ligne sur: http ://www.ditl.info/arttest/art7857.php. 